\title{
Tunable Gaussian mask for extending the depth of field
}

\author{
Jorge Ojeda-Castaneda, ${ }^{* 1}$ Emmanuel Yépez-Vidal, ${ }^{1}$ Eloy García-Almanza, ${ }^{1}$ and Cristina M. Gómez-Sarabia ${ }^{2}$ \\ ${ }^{1}$ Electronics Department, University of Guanajuato, Salamanca 36885, México, \\ ${ }^{2}$ Digital Arts, Department, University of Guanajuato, Salamanca 36885, México
}

Received September 01, 2012; accepted September 21, 2012; published September 30, 2012

\begin{abstract}
We present the use of two asymmetrical amplitude masks, for generating a tunable Gaussian filter. The two masks work as a pair. By introducing a lateral displacement, between the members of the pair, one can tune the width of the Gaussian filter. This tunable mask is useful for controlling unwanted oscillations in the MTFs, which are associated with the use of phase filters extending the depth of field.
\end{abstract}

One can extend the depth of field of an optical system, by a two-step process. In the first step, denoted as preprocessing, a spatial filter reduces the impact of focus errors on the modulation transfer function (MTF). In the second step, denoted as post-processing, a digital filter restores modulation losses on the gathered picture [1-2].

Certain amplitude masks and some phase filters are useful for producing out-of-focus MTFs that do not have zeros inside their passband [3-16]. However, the out-offocus MTFs exhibit undesired oscillations around a tendency line.

For reducing these spurious oscillations, on the MTFs, we proposed previously the use a Gaussian apodizer [17]. Here, we discuss an optical method for implementing a tuneable Gaussian filter, which can be used as an apodizer.

For the sake of clarity our initial model is 1-D. However, at the end of the paper we give the 2-D formulas. As in previous publications, we use the Greek letter $\mu$ for representing the spatial frequency variable; the Greek letter $\Omega$ denotes the cut-off spatial frequency of the pupil aperture. Now, we propose to use the following pair of amplitude masks. It is convenient to start our discussion, by considering the amplitude transmittance of the first mask as

$$
\mathrm{T}_{1}(\mu)=\mathrm{e}^{-\mathrm{a}\left[1+\left(\frac{\mu}{2 \Omega}\right)^{3}\right]} \operatorname{rect}\left(\frac{\mu}{4 \Omega}\right)
$$

In Eq. 1, the lower case letter "a" denotes dimensionless damping factors of the Gaussian function. From Eq. 1, we

\footnotetext{
*jorge_ojedacastaneda@yahoo.com
}

note that the length of the mask is $4 \Omega$; and that $\mathrm{T}_{1}(-2 \Omega)=1$, while $\mathrm{T}_{1}(2 \Omega)=\mathrm{e}^{-2 \mathrm{a}}$. The amplitude transmittance of the of the second mask is

$$
\mathrm{T}_{2}(\mu)=\mathrm{e}^{-\mathrm{a}\left[1-\left(\frac{\mu}{2 \Omega}\right)^{3}\right]} \operatorname{rect}\left(\frac{\mu}{4 \Omega}\right) .
$$

From Eq. 2, we recognize that the length of the mask is $4 \Omega$, and that $\mathrm{T}_{2}(-2 \Omega)=\mathrm{e}^{-2 \mathrm{a}}$, while $\mathrm{T}_{1}(2 \Omega)=1$. In Fig. 1 we depict schematically the two masks on top of the pupil aperture, which has a length equal to $2 \Omega$.

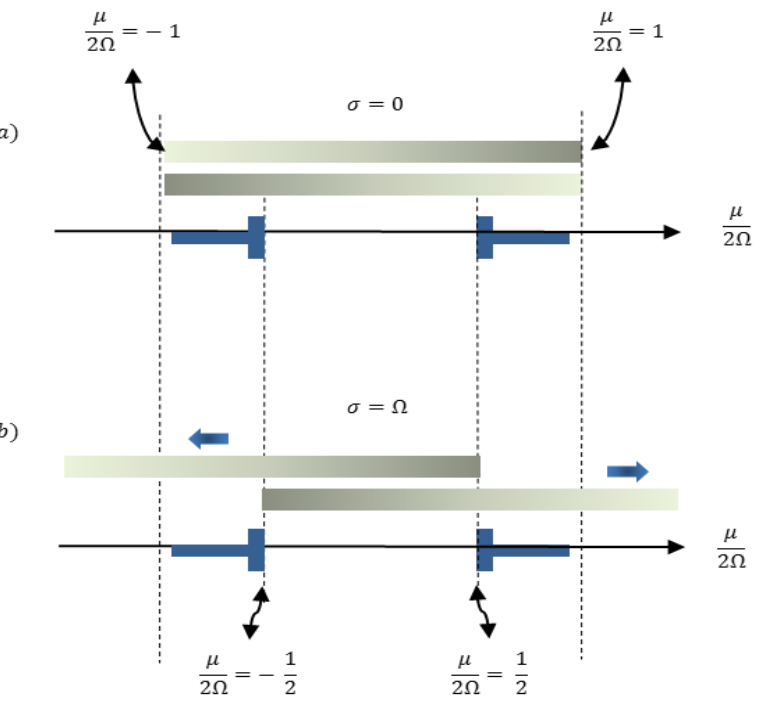

Fig. 1. Schematics of the two masks on the pupil aperture.

We introduce a lateral displacement, $2 \sigma$; between the masks. The parameter $\sigma$ varies from zero to $\Omega$. Hence, Fig. 1 depicts the two extreme cases. Next, we recognize that the overall amplitude transmittance inside the pupil aperture is

$$
\mathrm{T}(\mu ; \sigma)=\mathrm{T}_{1}(\mu+\sigma) \mathrm{T}_{2}(\mu-\sigma) \operatorname{rect}\left(\frac{\mu}{2 \Omega}\right) .
$$

By substituting Eqs. 1 and 2 in Eq 3, we obtain 


$$
\mathrm{T}(\mu ; \sigma)=\mathrm{e}^{-2 \mathrm{a}\left[1+\left(\frac{\sigma}{2 \Omega}\right)^{3}\right]} \mathrm{e}^{-\left(\frac{3 \mathrm{a} \sigma}{4 \Omega}\right)\left(\frac{\mu}{\Omega}\right)^{2}} \operatorname{rect}\left(\frac{\mu}{2 \Omega}\right)
$$

It is apparent from Eq. 4 that inside the pupil aperture, the overall amplitude transmittance varies as a Gaussian function. In Fig. 2, we depict the case $\sigma=0$. In this case, we obtain a uniform attenuating window.

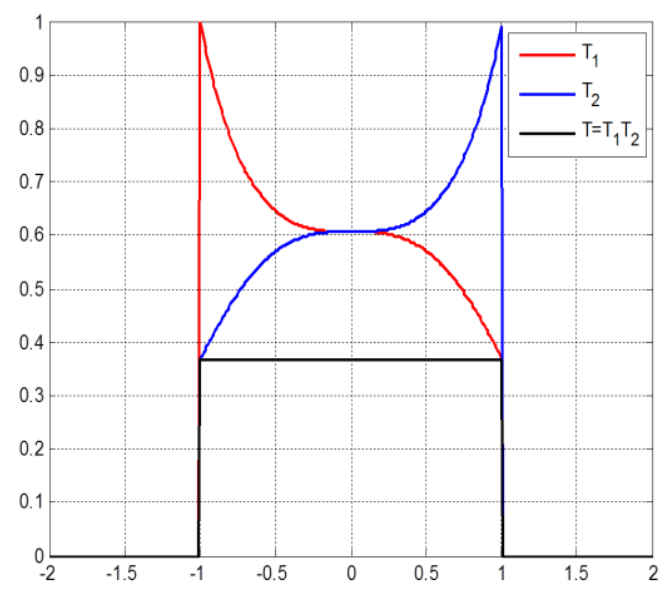

Fig. 2. Amplitude transmittance for $\sigma=0$.

In Fig. 3, we depict the amplitude transmittances associated to the case $\sigma>0$.

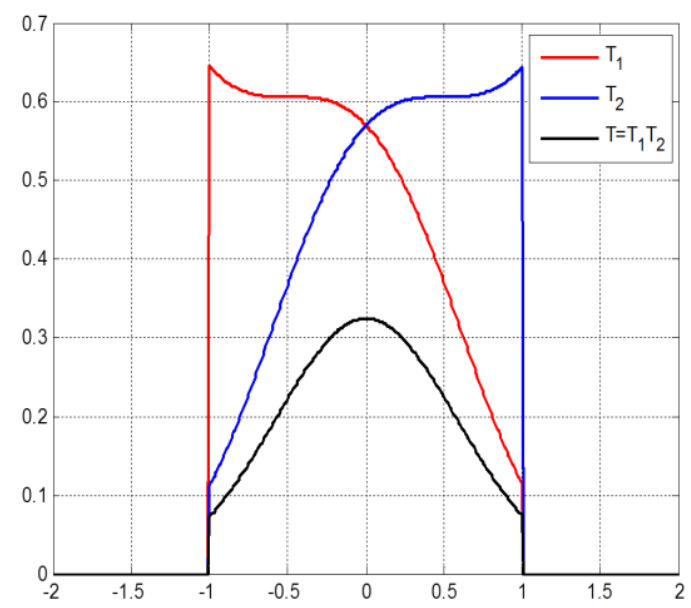

Fig. 3. Amplitude transmittance for $\sigma>0$.

For $\sigma \neq 0$, it is relevant to identify the parameter $\mu_{0}$, known as the half width at half maximum.

$$
\mu_{0}=2 \Omega \sqrt{\frac{\ln (2)}{3 \mathrm{a}\left(\frac{\sigma}{\Omega}\right)}} .
$$

Hence, for the maximum displacement $\sigma=\Omega$, the minimum value of $\mu_{0}$ is

$$
\mu_{0}=\sqrt{2 \ln (2)} \frac{\Omega}{\pi \mathrm{a}}
$$

Equation 6 is useful for setting the value of the dimensionless damping factor. For example, if $\mu_{0}=\Omega / 3$, then $a=12 \ln (2)$. Next, we recognize that the optical system suffers from focus errors. Then, generalized pupil function is

$$
\mathrm{P}(\mu ; \mathrm{W} ; \sigma)=\mathrm{T}(\mu ; \sigma) \mathrm{e}^{\mathrm{i} 2 \pi \mathrm{W}\left(\frac{\mu}{\Omega}\right)^{2}} \operatorname{rect}\left(\frac{\mu}{2 \Omega}\right) .
$$

In Eq. 7, the upper case letter $\mathrm{W}$ is a shorthand notation for representing the 1-D version of Hopkins focus error coefficient, $\mathrm{W}_{2,0}$, in units of wavelength $\lambda$. That is, $\mathrm{W}=$ $\left(\mathrm{W}_{2,0} / \lambda\right)$. See reference [18].

According to Ref. [19], all possible MTFs are suitable contained in the modulus of the ambiguity function of Eq. 7. In Fig. 4, we show a set of images that display the variations of the ambiguity function modulus.
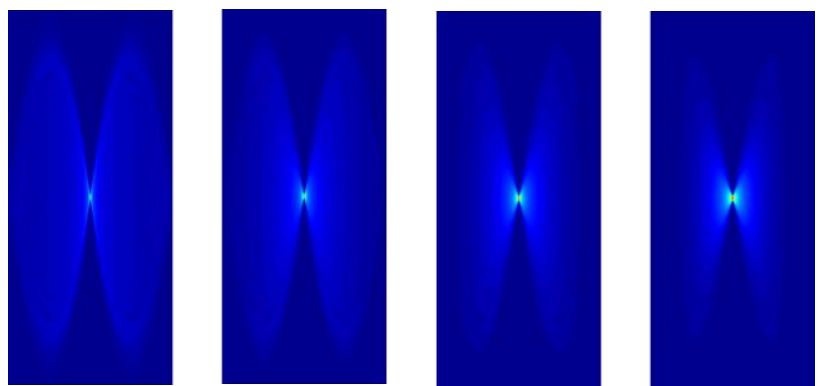

Fig. 4. Modulus of the ambiguity function.

For the numerical evaluation of Fig. 4, we use a cubic phase-mask [8], and the proposed tuneable Gaussian filter. The parameter $\sigma$ changes from zero to $3 \Omega / 4$, in steps of $\Omega / 4$. We note that the proposed filter reduces the spurious oscillations on the ambiguity function. This feature is emphasized in Fig. 5, which shows a radial scanning of the ambiguity function, for obtaining the MTFs, for $\sigma=0$, and $\sigma=3 \Omega / 4$. 


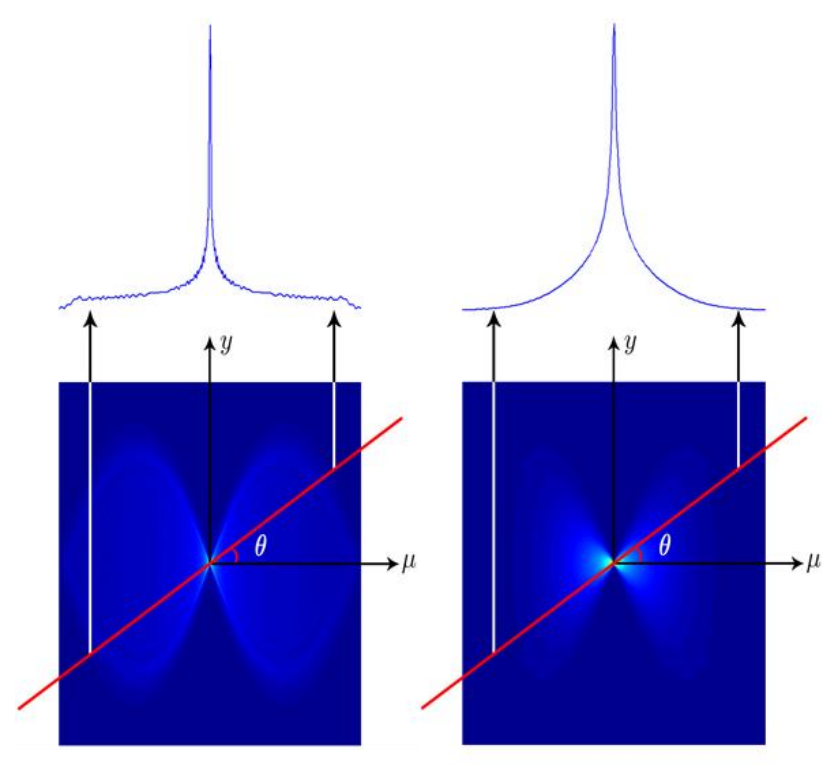

Fig. 5. Radial scanning of the ambiguity functions.

Finally, we report the 2-D version of the proposed filter. The amplitude transmittance of the first mask is

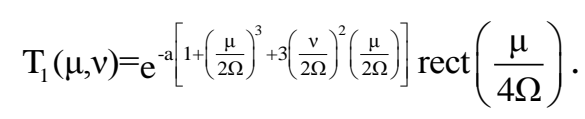

The amplitude transmittance of the second mask is

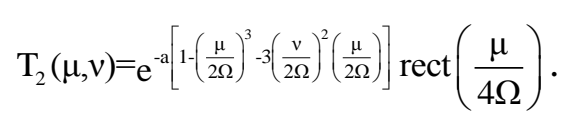

At the corners of the rectangular window, the 2-D masks have either maximum transmittance (equal to unity) or minimum transmittance [equal to $\exp (-8 \mathrm{a})$ ]. The amplitude transmittance of the proposed, 2-D filter is

$$
\mathrm{T}(\mu, v ; \sigma)=\mathrm{T}_{1}(\mu+\sigma, v) \mathrm{T}_{2}(\mu-\sigma, v) \operatorname{rect}\left(\frac{\mu}{2 \Omega}\right) \operatorname{rect}\left(\frac{v}{2 \Omega}\right)
$$

By substituting Eqs. 8 and 9 in Eq. 11, we obtain

$$
\mathrm{T}(\mu, v ; \sigma)=\mathrm{e}^{-2 \mathrm{a}\left[1+\left(\frac{\sigma}{2 \Omega}\right)^{3}\right]} \mathrm{e}^{-\left(\frac{3 \mathrm{a} \sigma}{4 \Omega}\right)\left[\left(\frac{\mu}{\Omega}\right)^{2}+\left(\frac{v}{\Omega}\right)^{2}\right]} \operatorname{rect}\left(\frac{\mu}{2 \Omega}\right) .
$$

It is apparent from Eq. 11 that the overall amplitude transmittance varies as a 2-D, Gaussian function inside a rectangular window.
Summarizing, we have presented the use of two amplitude masks, acting as a pair, for implementing a rectangular Gaussian filter. The width of the Gaussian function can be tuned by introducing a lateral shift between the members of the pair. We have reported analytical expressions for the 1-D version of the proposed filter; as well as analytical expressions for the 2-D version. We have indicated that it can be tuned to reduce the spurious oscillations on the MTF, which are produced by using phase filters that extend the depth of field.

We express our gratitude to CoNaCyT, México, for financial support, through the research grant 157276.

\section{References}

[1] G. Hauesler, Opt. Commun. 6, 38 (1972)

[2] J. Ojeda-Castañeda, R. Ramos, A. Noyola-Isgleas, Appl. Opt. 27, 2583 (1988).

[3] M. Mino, Y. Okano, Appl. Opt. 10, 2219 (1971).

[4] J. Ojeda-Castañeda, L.R. Berriel Valdos, E. L. Montes, Opt. Lett. 8(8), 458 (1983).

[5] J. Ojeda-Castañeda, L.R. Berriel-Valdos, E. Montes, Opt. Lett. 10(11), 520 (1985).

[6] J. Ojeda-Castañeda, A. Díaz, Appl. Opt. 27, 4163 (1988).

[7] J. Ojeda-Castañeda, L.R Berriel-Valdos, E. Montes, Appl. Opt. 27(4), 790 (1988).

[8] E.R. Dowski, T.W. Cathey, Appl. Opt. 34, 1859 (1995).

[9] H. Wang, F. Gan, Appl. Opt. 40, 5658 (2001).

[10] N. George, W. Chi, J. Opt. A: Pure Appl. Opt. 5, S157 (2003).

[11] A. Castro, J. Ojeda-Castañeda, Appl. Opt. 47(17), 1 (2004).

[12] Á. Sauceda-Carvajal, J. Ojeda-Castañeda, Opt. Lett. 29(6), 560 (2004).

[13] G. Mikula, Z. Jaroszewicz, A. Kolodziejczyk, K. Petelczyc, M. Sypek, Opt. Exp. 15, 9184 (2007).

[14] J. Ojeda-Castaneda, J.E.A. Landgrave, C.M. Gómez-Sarabia, Appl. Opt. 47, E99 - E105 (2008).

[15] J. Ares García, S. Bará, M. Gomez García, Z. Jaroszewicz, A. Kolodziejczyk, K. Petelczyc, Opt. Exp. 16, 18371 (2008).

[16] G. Muyo, A. Singh, M. Andersson, D. Huckridge, A. Wood, A.R. Harvey, Opt. Exp. 17(23), 21118 (2009).

[17] J. Ojeda-Castañeda, E. Yépez-Vidal, E. García-Almanza, Phot. Lett. Poland 2, 162 (2010).

[18] H.H. Hopkins, Wave Theory of Aberrations (Oxford, 1950).

[19] K.-H. Brenner, A.W. Lohmann, J. Ojeda-Castaneda, Opt. Comm. 77, 89 (1982). 